\title{
A Two-Dimensional Quadrupole Ion Trap Mass Spectrometer
}

\author{
Jae C. Schwartz and Michael W. Senko \\ LC and LC/MS Division, Thermo Finnigan, San Jose, CA, USA \\ John E. P. Syka \\ LC and LC/MS Division, Thermo Finnigan, Charlottesville, VA, USA
}

\begin{abstract}
The use of a linear or two-dimensional (2-D) quadrupole ion trap as a high performance mass spectrometer is demonstrated. Mass analysis is performed by ejecting ions out a slot in one of the rods using the mass selective instability mode of operation. Resonance ejection and excitation are utilized to enhance mass analysis and to allow isolation and activation of ions for $\mathrm{MS}^{\mathrm{n}}$ capability. Improved trapping efficiency and increased ion capacity are observed relative to a three-dimensional (3-D) ion trap with similar mass range. Mass resolution comparable to 3-D traps is readily achieved, including high resolution at slower scan rates, although adequate mechanical tolerance of the trap structure is a requirement. Additional advantages of 2-D over 3-D ion traps are also discussed and demonstrated. (J Am Soc Mass Spectrom 2002, 13, 659-669) (c) 2002 American Society for Mass Spectrometry
\end{abstract}

$\mathrm{T}$ Two dimensional (2-D) radio frequency multipole ion traps have been used for several years for the study of spectroscopic and other physical properties of ions [1,2]. The earliest application of 2-D multipole ion traps in mass spectrometry involved the use of the collision cell of a triple quadrupole instrument for studying ion-molecule reactions [3]. More recently, multipole ion traps have been used in mass spectrometers as part of hybrid systems including Fourier transform ion cyclotron resonance (FTICR) [4-7], time-of-flight (TOF) [8-11], and standard three-dimensional (3-D) ion trap mass spectrometers [12,13]. Several of the quadrupole based 2-D ion traps are capable of mass selective isolation and activation of ions including $\mathrm{MS}^{3}$ analysis [7,9-11].

Syka and Fies have described the theoretical advantages of 2-D versus 3-D quadrupole ion traps for Fourier transform mass spectrometry [14]. These advantages include reduced space charge effects due to the increased ion storage volume, and enhanced sensitivity for externally injected ions due to higher trapping efficiencies. Bier and Syka described several forms of linear and circular 2-D ion traps with larger ion capacity to be used as mass spectrometers [15] using the mass selective instability mode of operation [16] similar to that used in all commercial 3-D quadrupole ion trap instruments.

Published online April 26, 2002

Address reprint requests to Jae C. Schwartz, Thermo Finnigan, 355 River Oaks Parkway, San Jose, CA 95134, USA. E-mail: jschwartz@ thermofinnigan.com
Despite the previously described advantages and the recent progress with 2-D ion traps in hybrid instruments, only a few examples have appeared using these traps as stand-alone mass spectrometers. Senko et al. recently demonstrated image current detection with FT analysis in a 2-D ion trap which utilized independent detection electrodes between the quadrupole rods [17]. Although promising results were presented, space charge effects were found to limit this configuration's ultimate performance as the motion of the ions is largely restricted to one of the symmetry planes of the device. The relative insensitivity of the image current ion detection scheme precluded the use of lower numbers of trapped ions to avoid such space charge effects.

Welling et al. demonstrated two variations of mass selective instability in a stand-alone 2-D quadrupole ion trap [18]. The first method, referred to as " $q$-scanning", exploited the field penetration of the detector between the quadrupole rods to allow for ion extraction using a downward ramp of the RF voltage. This allowed relatively quick scanning of a broad mass range (1 second scan from $20-1000 \mathrm{~m} / \mathrm{z}$ ), but produced mass spectral resolution of only five to six. The second method, referred to as "secular scanning", used frequency swept parametric excitation to eject ions between the quadrupole rods. Although the scan rate was 100x slower than the q-scan, a resolution of 800 at $\mathrm{m} / \mathrm{z} 130$ was obtained.

Lammert et al. have presented preliminary results using a toroidal ion trap with mass selective instability [19]. Although this trap did not use the linear geometry of traditional 2-D ion traps, the quadrupolar trapping potential was two dimensional in nature, and did 


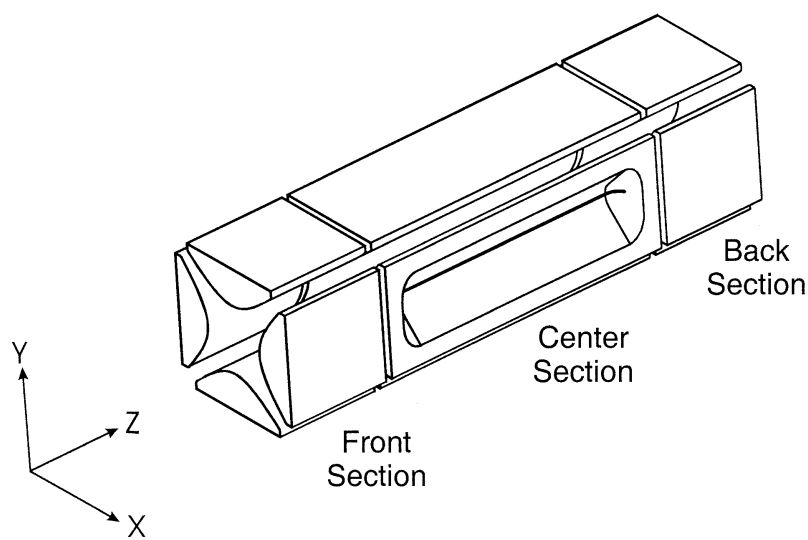

Figure 1. Basic design of the two-dimensional linear ion trap.

provide the benefit of increased ion storage capacity. Recently, Hager has demonstrated a novel ion trapping mode of operation on a modified triple quadruopole [20]. Fringe field effects caused by lenses at the ends of the quadrupole, normally considered detrimental, are exploited to eject trapped ions axially in a mass dependent fashion.

Here we apply many of the ideas for mass spectral analysis with 2-D quadrupole ion traps previously described by this laboratory $[14,15]$. The 2-D ion trap described here is capable of operating in a manner analogous to a conventional 3-D ion trap, but also demonstrates distinct and unique operational characteristics. Experimental data is provided to support the predicted advantages of an increased ion storage capacity and enhanced trapping efficiency of externally injected ions. General performance and capabilities of the instrumental configuration are demonstrated along with other unique advantages of this type of mass spectrometer.

\section{Experimental}

The basic design of the 2-D ion trap used in this work is depicted in Figure 1. The quadrupole structure has hyperbolic rod profiles with an $\mathrm{r}_{0}$ of $4 \mathrm{~mm}$, similar to that used in the Finnigan TSQ 700 series triple quadrupole instrument, but each rod is cut into three axial sections of 12, 37, and $12 \mathrm{~mm}$ length. The three sections, each with a discreet DC level, allow containment of the ions along the axis in the central section of the device, avoiding any possible fringe field distortions to the trapping and resonance excitation fields.

The ability to avoid fringe field distortions for the dipole resonance excitation field is shown in Figure 2a. All three sections of the top rod have $1 \mathrm{~V}$ applied, and all three sections of the bottom rod have $-1 \mathrm{~V}$ applied. Application of appropriate potentials to the end sections restricts ions to the center section of the trap, where the dipole field has no axial component. For the single section trap shown in Figure 2b, the axial trapping potential has to be created by voltages on the end

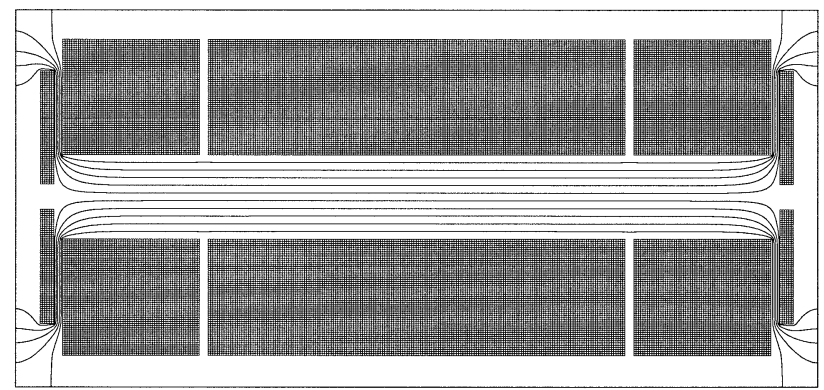

(a)

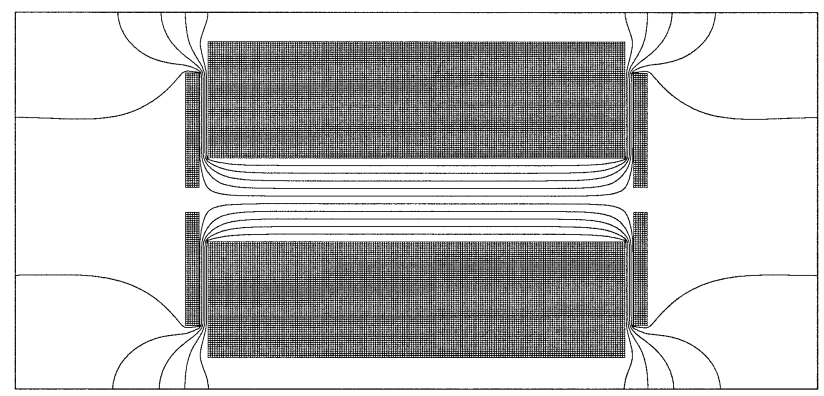

(b)

Figure 2. Simion simulation of the resonance excitation field comparing (a) a three section device and (b) a single section device with end plates for axial trapping.

lenses. As shown, the radial dipole field has a significant axial component in the region near the end lenses. In this region, ions will experience some axial excitation, which could lead to unintended axial ejection. This approach to avoiding fringe field effects is similar to that utilized in FTICR cell design for reducing undesirable axial ejection [21]. A similar distortion also occurs to the quadrupolar trapping potential at the end of the single section device. This distortion has recently been exploited to generate spectra through mass selective axial ejection [20].

A $0.25 \mathrm{~mm}$ high slot was cut along the middle $30 \mathrm{~mm}$ of the center section of one rod for ion ejection. To help compensate for the detrimental field effects due to the presence of the slot, the rod with the slot and the rod opposite it (the $\mathrm{X}$ rods) were moved out from the center $0.75 \mathrm{~mm}$ beyond their normal position. This appears to have analogous effects to the "stretch" in most commercial 3D ion traps [22] and is the subject of ongoing investigation and optimization. The length of the center section was limited so that the exiting beam of ions would be collected by the standard detection system of a commercial Thermo Finnigan LCQ 3-D (San Jose, California) ion trap. This detector system includes a conversion dynode held at $-15 \mathrm{kV}$ (for positive ions) and a channeltron electron multiplier. In order to assure that ions are efficiently focused onto the detector, the ion optics simulation program Simion was used to examine the trajectories of ions exiting the 2-D ion trap. Examples are shown in Figure 3 where $100 \mathrm{eV}$ and 10 $\mathrm{keV}$ ions [23], distributed along the entire length of the slot, are all focussed onto the $-15 \mathrm{kV}$ dynode cup. 


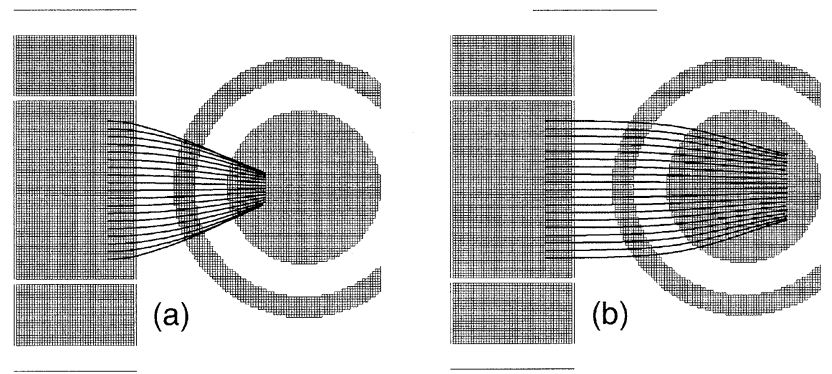

Figure 3. (a) Simion simulation of $100 \mathrm{eV}$ ions exiting the 2-D trap along the length of the exit slot and being focussed onto the $-15 \mathrm{kV}$ conversion dynode. (b) $10 \mathrm{keV}$ ions simulated.

Figure 4 shows all the voltages necessary to operate the 2-D ion trap as a mass spectrometer. These voltages include three DC voltages $( \pm 100 \mathrm{~V})$ applied to the separate sections of each rod to produce an axial trapping field, two phases of the primary RF voltage ( $\pm 5 \mathrm{kV}$ rod to ground, $1 \mathrm{MHz}$ ) applied to the rod pairs to produce the radial trapping field, and two phases of supplemental AC voltage $( \pm 80 \mathrm{~V}, 5-500 \mathrm{kHz})$ applied across the $\mathrm{X}$ rods for isolation, activation, and ejection of
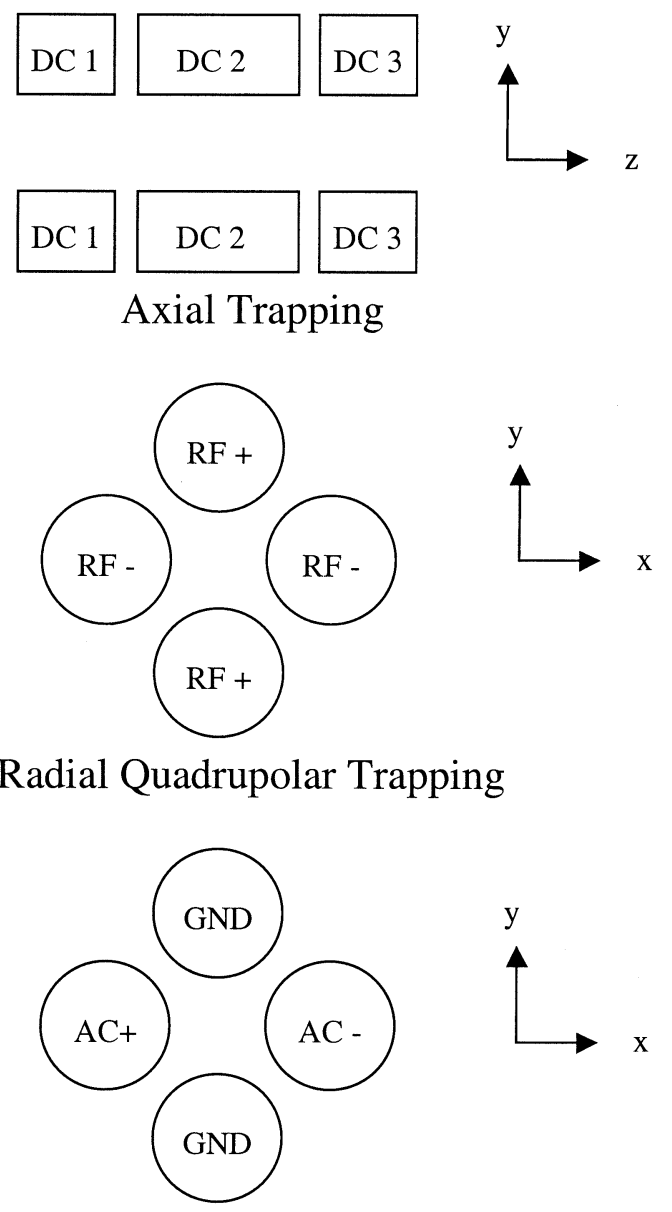

\section{Radial Dipolar Excitation}

Figure 4. Scheme for application of DC, RF trapping, and AC excitation voltages necessary for operation of the 2-D ion trap. ions. For the RF and AC signals applied to each rod, the voltages need to be applied equally across all segments of the rod to minimize any unintended axial fields. The appropriate combination of the above potentials results in nine separate voltages applied to the twelve electrodes. In initial experiments, the RF electronic system of an LCQ was adapted to apply the main RF voltage to only the $\mathrm{Y}$ quadrupole rods. A tri-filar (three-wire) RF transformer coil was constructed which allowed the coupling of three DC voltages for the three axial sections of the $\mathrm{Y}$ rod set with the main quadrupolar RF. The resonance excitation voltage was then easily applied across the $X$ rods without having it combined with the RF voltage. The only modification was that two extra secondary windings were added to the AC output transformer of the LCQ to allow coupling on to the offset voltages for the three $X$ rod end segments. This mode of operation was analogous to that of a conventional 3-D ion trap, with a single phase of RF voltage applied to the ring electrode, and the resonance excitation voltage applied across the end caps. This simple approach generated mass spectra. However, the trapping efficiency for externally injected ions was limited due to the strong $\mathrm{Z}$ axis RF potential gradients at the entrance to the device caused by the asymmetrical application of RF voltage to the electrodes.

To maximize trapping efficiencies for axially-injected ions, a balanced RF system was constructed which symmetrically applied opposite phases of the RF voltage to the $X$ and $Y$ rod sets. Obtaining the required nine different superpositions of the supplemental AC voltage, the three DC offset voltages and the trapping RF voltage required construction of a considerably more elaborate RF/AC/DC system. The conceptually simple approach using a broadband transformer to couple the AC voltage on to the high RF voltages generated by the tuned circuit transformer has been found to be adequate only at the relatively low RF voltages previously utilized ( $\sim 600 \mathrm{~V}$ peak rod-to-ground for $1 \mathrm{MHz}, 4 \mathrm{~mm}$ $\mathrm{r}_{0}, m / z 587$ at $\mathrm{q}=0.623$ ) [10]. At the full voltage of this RF system ( $5 \mathrm{kV}$ peak rod-to-ground), the level of isolation across the supplemental transformer that was necessary to prevent breakdown created very uneven coupling of broadband AC signals. To avoid these isolation problems, all voltages were coupled directly through the center-tap of the RF coil on independent filars. LCQ RF regulation circuitry, which was designed to produce and control only a single phase of RF voltage, was used without modification. Ideally the RF voltage feedback for the servo control system that provides the required precise control of the RF voltage amplitude applied between pairs of rods should be sampled from both ends of the transformer coil ( $\mathrm{X}$ and $\mathrm{Y}$ phases). However, sufficient RF amplitude stability for this investigation was achieved by detecting only the $\mathrm{Y}$ phase of the RF voltage for feedback. A complete description of the circuit will be the subject of a future report.

Resonance excitation was utilized for isolation, activation and mass analysis. Isolation of ions was achieved 


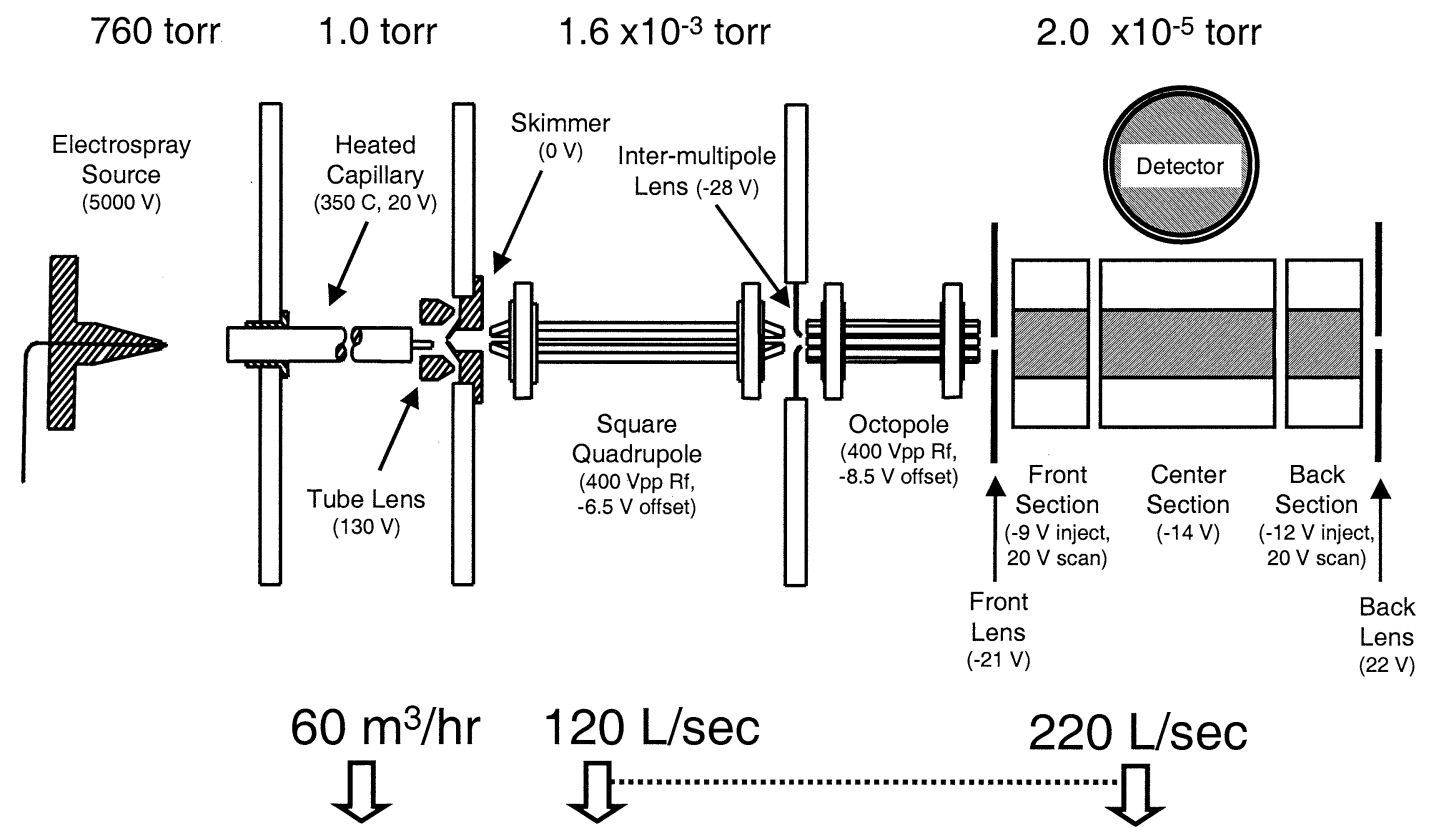

Figure 5. The overall instrument configuration along with typical operating voltages and pressures.

using resonance ejection of all unwanted ions with a $5-500 \mathrm{kHz}$ multi-frequency waveform consisting of sine components spaced every $0.5 \mathrm{kHz}$ [24]. Sine components were removed from the isolation waveform at the oscillation frequency of the ion of interest. The ion to be isolated was typically placed at a $\mathrm{q}$ of 0.83 , which corresponded to a frequency of $\sim 368 \mathrm{kHz}$. The isolation waveform was applied for 16 milliseconds at an amplitude adjusted to assure ejection of all other ions throughout the mass range.

Activation of ions was accomplished with a single frequency, low amplitude $(\sim 1 \mathrm{~V})$ resonance excitation signal applied for 30 milliseconds at a frequency corresponding to an activation q of $0.25-0.35$. Mass selective instability with resonance ejection at a $\mathrm{q}$ of 0.88 was performed to generate mass spectra. The resonance ejection amplitude was scanned with $\mathrm{m} / \mathrm{z}$, using an intercept of $3 \mathrm{~V}$ and a slope of $20 \mathrm{mV} /(\mathrm{m} / \mathrm{z})$. Resonance ejection enhances resolution and sensitivity, as in 3-D ion traps [25]. Without resonance ejection, or with ejection at a $\mathrm{q}>0.88$, ion motion may grow in the $\mathrm{Y}$ direction, resulting in reduced ejection efficiency through the $0.25 \mathrm{~mm}$ slot.

Plate lenses were placed on the front and back of the trap structure to provide conductance limits. The aperture size for these lenses was $2 \mathrm{~mm}$. The trap was filled with helium to approximately three millitorr for all experiments [16]. The trap was mounted in a custom vacuum manifold with ion optics from a Thermo Finnigan LCQ Deca. The only modification was the lengthening of the square quadrupole in the second vacuum stage an additional $50 \mathrm{~mm}$ to span the larger chamber of the custom manifold. Three stages of differential pumping were provided by two Edwards (Nashua, NH)
E2M30 mechanical pumps in tandem, and one Pfeiffer (Wilmington, MA) TMH261/130 dual flow turbo pump. The overall instrument configuration is shown in Figure 5. Typical pressures and operational settings were as indicated. The system has both electrospray (ESI) and atmospheric pressure chemical ionization capabilities, however only ESI was used for this work.

The standard LCQ calibration mixture was used to evaluate the system, which consisted of Ultramark 1621 (PCR, Inc., Gainesville, FL) [26], caffeine and the peptide MRFA. The solution consisted of approximately $500 \mathrm{ng} / \mathrm{ul}$ of UM 1621, $6 \mathrm{ng} / \mathrm{ul} \mathrm{MRFA}$ and $40 \mathrm{ng} / \mathrm{ul}$ of caffeine in $50 \%$ acetonitrile, $25 \%$ water, $25 \%$ methanol with $.1 \%$ acetic acid. Alprazolam (Sigma-Aldrich, St. Louis, MO) was used for limit of detection measurements.

\section{Results and Discussion}

Various aspects of the performance of the 2-D ion trap were investigated and the results are discussed below. Comparison in most areas is made to a standard Thermo Finnigan LCQ Deca 3-D ion trap.

\section{Space Charge Limit}

Space charge effects can significantly limit the performance of all ion trap type mass spectrometers. These effects limit most aspects of instrument performance including resolution, mass accuracy, sensitivity and dynamic range. There are several types of space charge limits which are of practical importance [27].

Storage Space Charge Limit-the maximum number of ions which can be stored 
Activation Space Charge Limit-the maximum number of ions which can be stored while maintaining the ability to activate ions with a specified fragmentation efficiency

Isolation Space Charge Limit-the maximum number of ions which can be stored while maintaining the ability to isolate ions with a specified resolution and efficiency

Spectral Space Charge Limit-the maximum number of ions which can be stored while maintaining the ability to obtain a mass spectrum of some specified resolution and mass accuracy.

It is important to know that each space charge limit relates to a different ion capacity. The relative order of the different limits has generally been found to be:

Storage Limit $>$ Activation Limit $>$ Isolation Limit $>$ Spectral Limit

The different limits may vary by orders of magnitude and each depends on the device properties such as operating frequency and physical size. These values are also dependent on the particular mode of operation for the device such as the method of mass analysis or ion isolation.

The most critical limit is the spectral space charge limit which for typical operating methods is significantly lower than the storage limit by several orders of magnitude. That is, most traps can hold many more ions than typically can be used during generation of a mass spectrum having acceptable resolution and mass accuracy. This minimal spectral space charge limit constrains the signal-to-noise ratio for a single mass spectral scan.

Most space charge models $[1,10,12,28]$ consider only ions of a single mass trapped by a static electric field using the pseudo-potential approximation $(\mathrm{q}<$ 0.4 ), and are therefore of questionable utility when attempting to compare the spectral space charge limits of 2-D and 3-D traps for a mixture of ions ejected at a $\mathrm{q} \sim 0.9$. The simple model of Campbell et al. [10], which considers only the trapping volume, predicts the increased storage capacity for a 2-D trap to be:

$$
N_{2 D} / N_{3 D}=r_{0}{ }^{2} l / z_{0}^{3}
$$

Comparing a standard LCQ $\left(\mathrm{z}_{0}=7.07 \mathrm{~mm}\right)$ to this 2-D trap $\left(r_{0}=4 \mathrm{~mm}\right.$, effective length $\left.1=30 \mathrm{~mm}\right)$, the space charge limit should increase only 1.4x. The earlier model of Douglas [12] accounts for the different operating frequencies of the LCQ Deca $(760 \mathrm{kHz})$ and this 2-D trap $(1 \mathrm{MHz})$ and predicts the space charge limit should increase 2.9x. The model of Prestage et al. [1] predicts the difference in ion capacity based upon equivalent micro-motion induced second-order Doppler shifts from the photo emission of trapped ions. This approach predicts the relative storage capacities of 2-D to 3-D traps to be:

$$
N_{2 D} / N_{3 D}=3 / 5\left(l / R_{s p h}\right)
$$

where $R_{s p h}$ is the radius of the uniform spherical ion cloud in a 3-D trap, and $l$ is the length of the uniform cylindrical ion cloud in a 2-D trap. The ion traps are assumed to be operated at the same trapping field frequency and operated such that trapped ions have identical characteristic frequencies of motion in each dimension of the quadrupole field. Using an estimated ion cloud radius of $1.0 \mathrm{~mm}$ from ion tomography [29] and an effective cloud length of $30 \mathrm{~mm}$, the 2-D trap used in our work should have a 18x higher space charge limit.

We have attempted to derive the relative ion capacities of ion traps when used for mass spectrometry purposes. The full derivation of the estimates for relative space charge capacities will be the subject of a future publication. In this derivation, the relative quantities of stored charge required to produce the same maximum percent error in the RF electric field are estimated. For simplicity of calculation, the trapped ion clouds are treated as having uniform density and either spherical (3-D) or cylindrical (2-D) shape. Unlike prior treatments, the ion clouds may be heterogeneous in $\mathrm{m} / \mathrm{z}$, and the pseudo-potential approximation is not invoked, as it is rarely applicable at the operating points used for mass analysis. These modifications require that the radii of the trapped ion clouds be estimated from experimental results [29].

The resulting expression for the relative ion capacity is proportional to the ratio of the ion cloud volume, and is given as:

$$
N_{2 \mathrm{D}} / N_{3 \mathrm{D}}=1 / 2\left(R_{2 \mathrm{D}}{ }^{2} l / R_{3 \mathrm{D}}^{3}\right)
$$

Assuming the two traps are operated identically (the same trapping field frequency and amplitude, collision gas pressure, etc.), the cloud radii should be identical. This results in the following simplification:

$$
N_{2 \mathrm{D}} / N_{3 \mathrm{D}}=1 / 2\left(l / R_{3 \mathrm{D}}\right)
$$

Using an estimated ion cloud radius $R_{3 D}$ of $1.0 \mathrm{~mm}$ from ion tomography performed with a 3-D trap [29] and a length $l$ of $30 \mathrm{~mm}$, the 2-D trap used for this work should have an approximately $15 x$ higher ion capacity than the comparable 3-D trap.

Figure 6a shows an experimental comparison of the spectral space charge limit for this 2-D ion trap and the standard 3-D trap. The space charge induced mass shift for the monoisotopic peak of MRFA $(\mathrm{m} / \mathrm{z} 524.3)$ is plotted against the measured total ion current (TIC) for an isolated window from $\mathrm{m} / \mathrm{z} 519.3$ to 529.3 . The chart clearly shows the significantly reduced shifting (approximately $20 \mathrm{x}$ ) for the 2-D trap relative to the standard 3-D trap.

The charge density can be shown to be somewhat controlled by changing the axial trapping potentials before scanning the ions out in order to restrict the axial spread of the ion cloud. Figure $6 \mathrm{~b}$ shows that the slope of the mass error versus TIC curves varies with the axial 

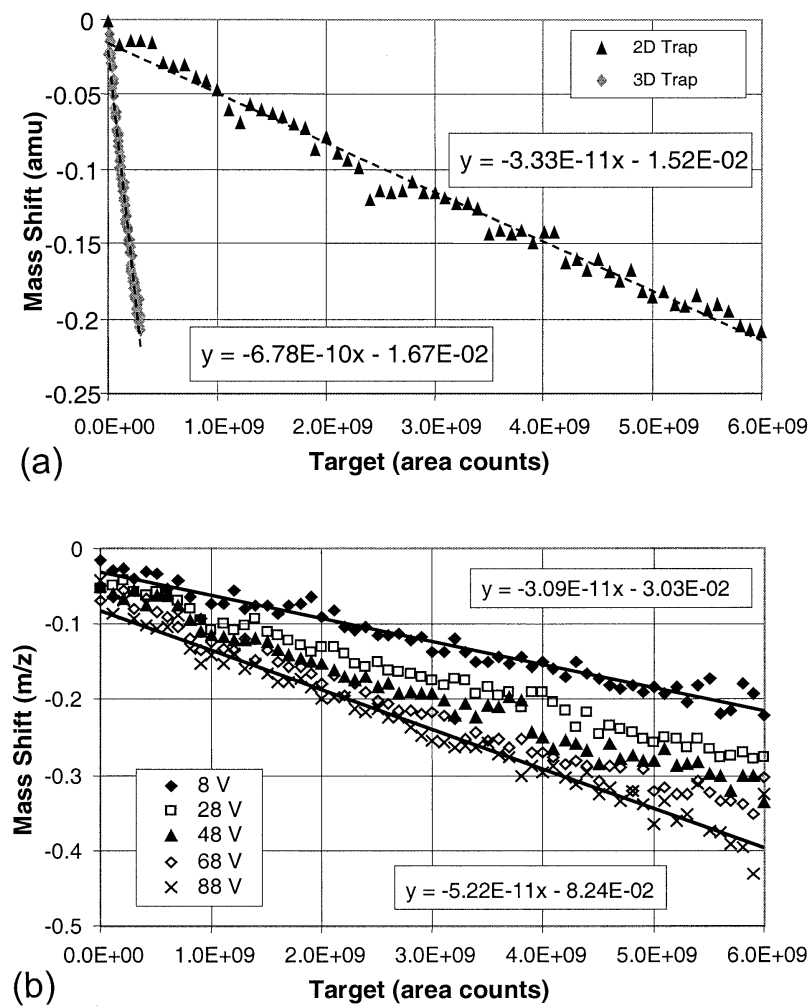

Figure 6. (a) Spectral space charge limit comparison between 2-D and 3-D ion traps. (b) The effect of the axial trapping potential on the spectral space charge limit for the 2-D trap.

trapping potential. This indicates that the range of axial trapping potentials studied here can affect the spectral space charge limit by approximately a factor of two. In order to maximize the spectral space charge limit, the axial trapping potentials should be adjusted to a minimum value which is just sufficient to maintain the ions in a position that allows ejection through the exit slot.

\section{Resolution}

Mass spectra are generated by the method of mass selective instability with resonance ejection; therefore resolution in the 2-D ion trap is controlled by many of the same processes as in a 3-D ion trap. However, unlike in a 3-D ion trap where the trap structure does not require high mechanical tolerances, the 2-D ion trap is far more susceptible to mechanical errors. The ion ejection process in the 3-D trap restricts the ions radially to approximately the center $1 \mathrm{~mm}$, limiting the overall volume and field inhomogeneities that the ions experience. At low axial trap potentials, the ions in this 2-D trap spread out across $\sim 30 \mathrm{~mm}$ in the axial direction, thus one can imagine if the quadrupole rods are not completely parallel, then ions at different axial positions will experience slightly different field strengths and have slightly different $q$ values. The variation in $q$ will cause ejection times that are dependent on ion axial position, which will degrade resolution.

As mentioned above, the axial dispersion and posi-
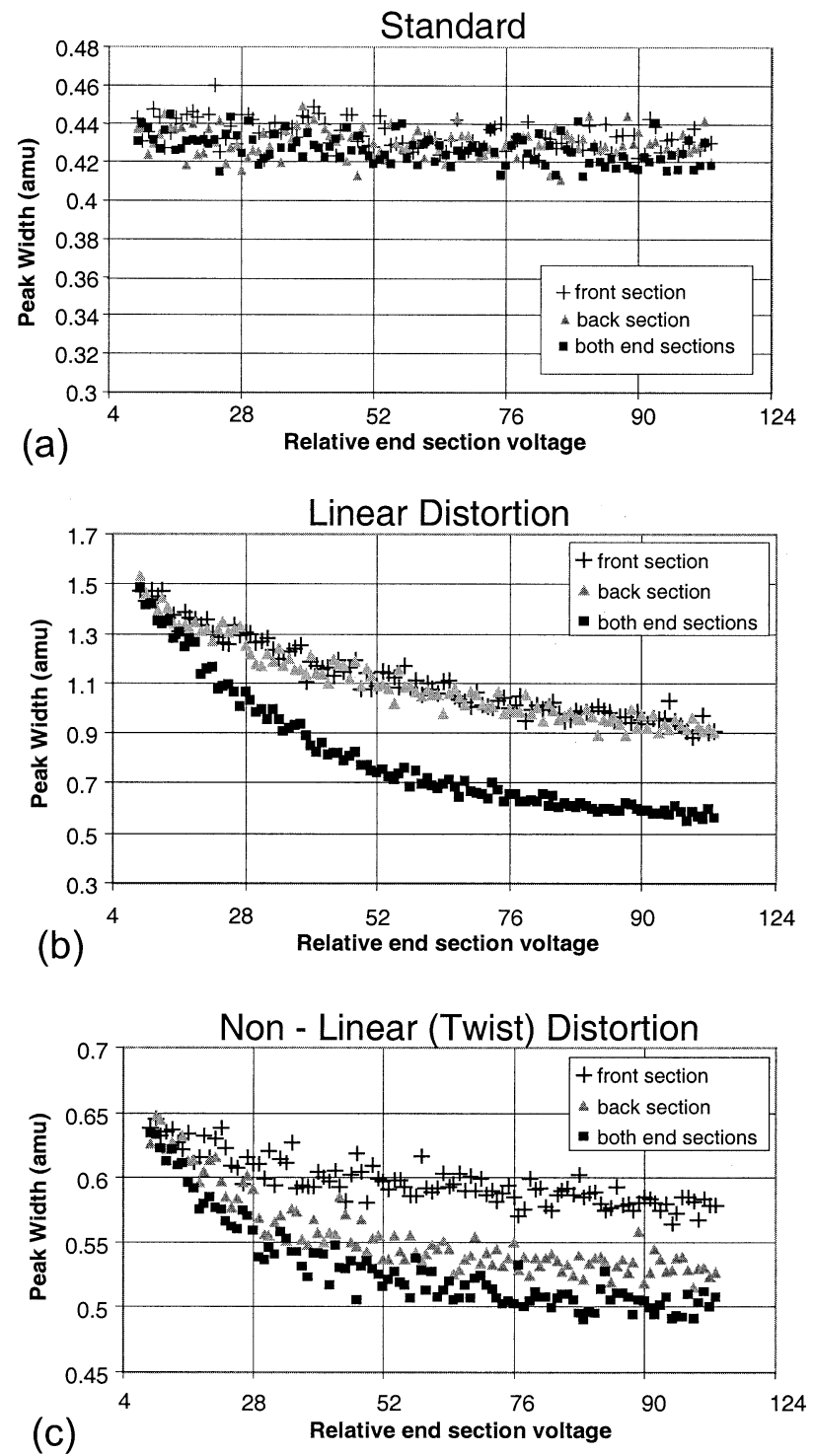

Figure 7. Effect of axial trapping potential on peak width for Ultramark at $\mathrm{m} / \mathrm{z} 1522$ for (a) a standard trap, (b) a linearlydistorted trap by slanting a rod, and (c) a non-linearly distorted trap by twisting a rod. Sections not scanned are held at $3 \mathrm{~V}$ relative to the center section.

tion of the ion cloud can be controlled by the axial trapping potentials during ejection. For a device with poor mechanical tolerances, the mass spectral resolution will vary with axial dispersion and position of the ion cloud due to variations in the strength of the trapping field. Figures $7 \mathrm{a}$ and $7 \mathrm{~b}$ show this effect on the Ultramark peak at $m / z 1522$ for both an unmodified 2-D ion trap and one which has been modified by placing a $0.125 \mathrm{~mm}$ shim on one end of one $X$ rod to create a deliberate linear variation of $r_{0}$ with axial position. Shimming of an $\mathrm{X}$ rod provides the worst case distortion, because both the trapping and radial excitation fields are axially inhomogeneous. For the unmodified trap, the peak width is relatively independent of the axial dispersion and position of the ion cloud. For the distorted trap, resolution is highly dependent on the 


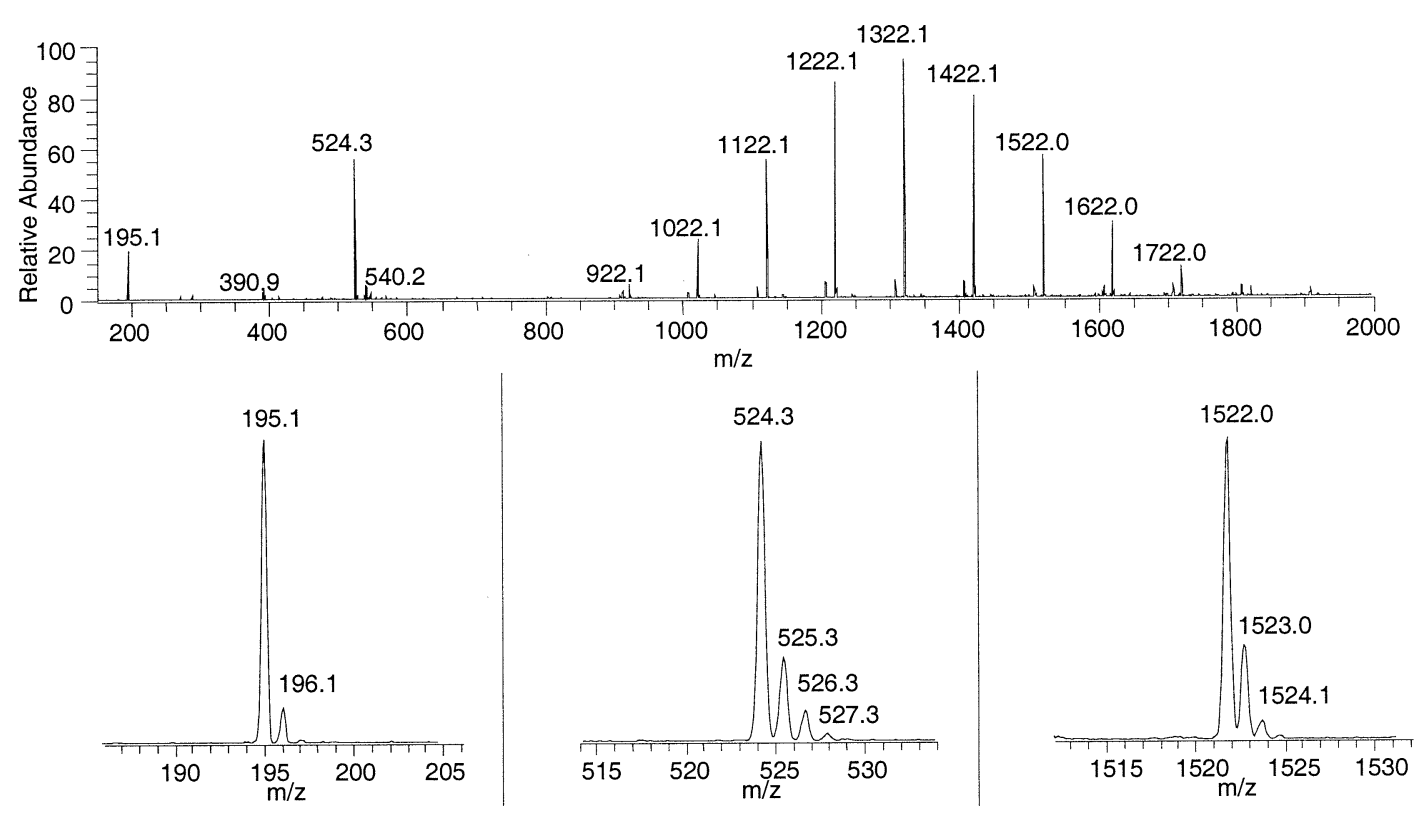

Figure 8. Full scan mass spectrum of the calibration mixture containing caffeine $(\mathrm{m} / \mathrm{z} 195)$, the peptide MRFA $(m / z 524)$, and Ultramark 1621 acquired at $5555 \mathrm{Da} / \mathrm{sec}$ with a $3 \times 10^{9}$ target TIC.

axial dispersion of the ion cloud, as shown by peak widths that are minimized when the axial potentials are maximized. This type of experiment can be used as a general method of evaluating the mechanical tolerances of the trap and can detect both linear distortions such as non-parallelism, or non-linear distortions such as a bent or twisted rod. A non-linear distortion such as a twisted rod will show a different variation in resolution when the ion cloud is biased axially from one side of the device versus the other. This effect is demonstrated in Figure $7 \mathrm{c}$, where a deliberate twist was created for one rod by placing a $0.125 \mathrm{~mm}$ shim on one corner of one rod, and thus the resolution varies differently when scanning the potential on the front section versus the back section.

For a device of limited mechanical tolerance, this leads to a compromise of either enhanced resolution with high axial trapping potentials and an axially restricted ion cloud, or an enhanced spectral space charge limit with low axial trapping potentials and an axially expanded ion cloud. The mechanical tolerances of the device used here are approximately $\pm 20 \mathrm{um}$. This was found to be sufficient to obtain unit resolution up to $\mathrm{m} / \mathrm{z} 2000$ when scanning at $5555 \mathrm{Da} / \mathrm{sec}$ with a $20 \mathrm{~V}$ difference between the center and end sections (Figure 8). This resolution is comparable to conventional 3-D ion traps of the same mass range.

As in the 3-D trap, higher resolution mass spectra can be obtained in the 2-D trap by reduction of the scan rate and resonance ejection amplitude [30]. Higher resolution scans have lower spectral space charge limits [27], which typically necessitates some signal averaging on 3-D traps. The increased ion capacity of the 2-D trap allows acquisition of high quality spectra with a single scan. Figure 9 shows example data of single scans for three different charge states of melittin at a scan rate of $278 \mathrm{Da} / \mathrm{sec}$. The total number of ions in each of these scans was approximately ten times higher than that normally used for an LCQ Deca. The variation in isotopic peak heights is attributed mainly to statistical fluctuations, since each displayed spectrum is the result of less than 500 detected ions.

\section{Ion Isolation, Activation, and $\mathbf{M S}^{\mathbf{n}}$}

Ion isolation is performed by the same method as used on the LCQ Deca [24], and provides equivalent results. The ability to isolate the ${ }^{13} \mathrm{C}$ isotope of MRFA at $\mathrm{m} / \mathrm{z}$ 525.3 from the ${ }^{12} \mathrm{C}$ and ${ }^{13} \mathrm{C}_{2}$ with $>95 \%$ efficiency is demonstrated in Figure 10. The $500 \mathrm{~Hz}$ spacing of the isolation waveform limits unit resolution isolation to ions below $\mathrm{m} / \mathrm{z} \sim 1500$. Above this $\mathrm{m} / \mathrm{z}$, the effective width of a $500 \mathrm{~Hz}$ isolation notch is greater than one $\mathrm{m} / \mathrm{z}$, and thus neighboring interferences may not be completely ejected. Other methods analogous to that used for 3-D ion traps can be implemented to yield higher resolution isolation [31]. In contrast to previous reports [11], no variation in isolation resolution or efficiency was observed across a broad range of helium pressures ( 0.5 to 5 millitorr). The difference is most likely due to the use of helium for a collision gas in this work, versus nitrogen used in the previous report.

Activation of isolated ions is performed using resonance excitation at a $\mathrm{q}$ of 0.25 . This $\mathrm{q}$ value is a compromise between yielding sufficient energy in the collisions to produce efficient fragmentation and maintaining fragment ion mass range, since a low mass cut off is established by the activation q. The MS/MS spectrum for the ${ }^{12} \mathrm{C}$ isotope of MRFA $(\mathrm{m} / \mathrm{z} 524.3)$ is shown in Figure 11. $\mathrm{MS}^{\mathrm{n}}$ can be achieved by simply 


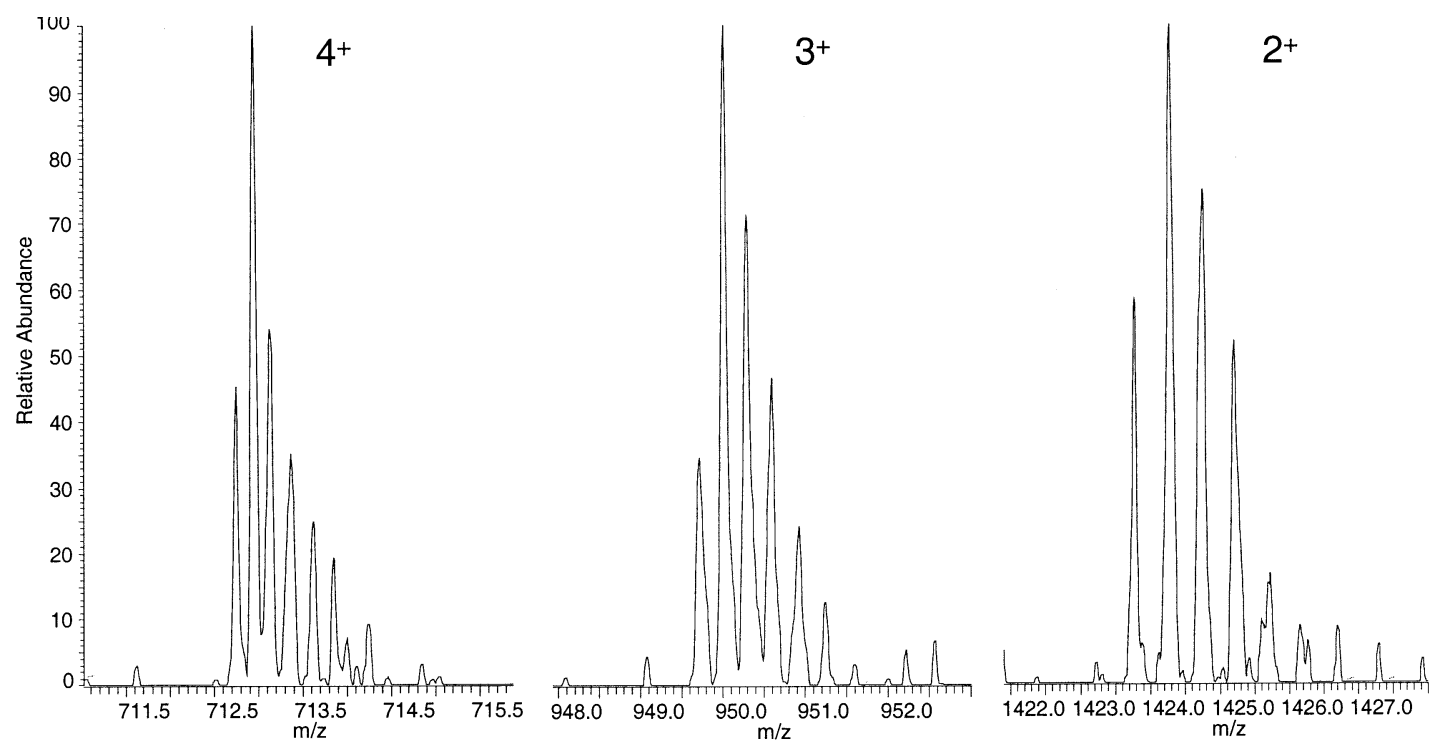

Figure 9. High resolution single scan spectra for Melittin acquired at $267 \mathrm{Da} / \mathrm{sec}$, demonstrating resolution of multiply-charged isotopes.

repeating the isolation and activation cycles. The dissociation efficiency for Figure 11 is $74 \%$. This efficiency is calculated by measuring the total area for fragment peaks and dividing by the total area of the isolated parent peak without activation. This efficiency is typical for many compounds examined, and is quite similar to the efficiency obtained using a 3-D trap.

\section{Mass Discrimination, Trapping Efficiency and Sensitivity}

Significant loss of ions and mass discrimination occurs in a 3-D ion trap due to the fact that upon injection from an external source, the ions must penetrate a significant RF field which exists along the axis of ion injection [32, 33]. Consequently, ions of a particular energy are successfully stored only when the correct amplitude and phase of the RF voltage exists. At all other combinations of phases and amplitudes, ions will have either too little or too much momentum such that their trajectory within the trap is not lengthy enough to allow an adequate number of collisions with the buffer gas to remove sufficient kinetic energy for ions to be trapped. This results in significant discrimination when attempting to trap a wide range of $m / z^{\prime} \mathrm{s}$. In the 2-D trap, the RF field in the direction of ion injection is minimized.

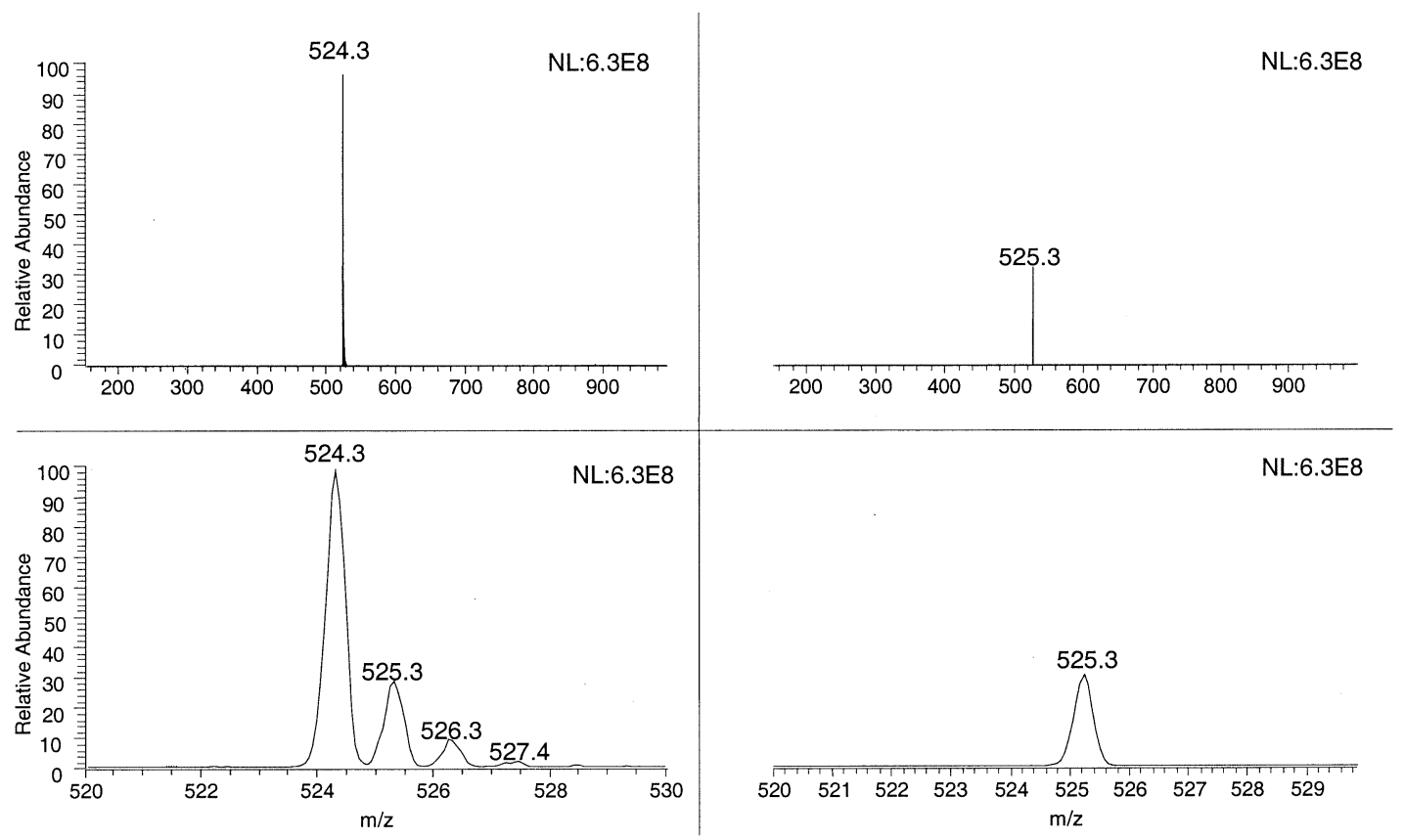

Figure 10. Isolation of ${ }^{13} \mathrm{C}$ isotope of peptide MRFA at $m / z 525.3$ with high efficiency. 


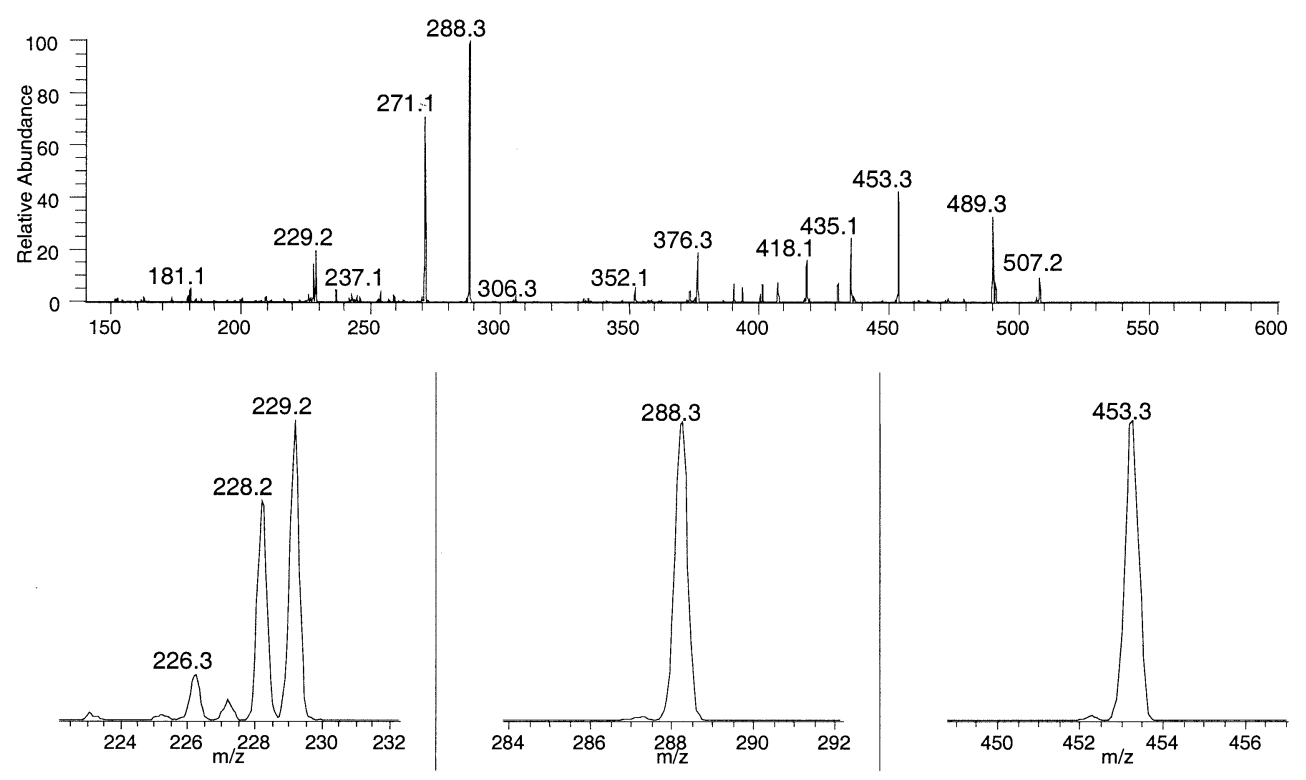

Figure 11. MS/MS of peptide MRFA at $\mathrm{m} / \mathrm{z} 524.3$ with $74 \%$ fragmentation efficiency.

Theoretically, matched RF amplitudes applied out of phase to both sets of rods leaves only the DC offset potential along the ion injection axis. Practically, misbalance of the amplitudes of the different RF phases or unequal spacing of the rod sets can still cause ion losses. Here, the $0.75 \mathrm{~mm}$ stretch of the $\mathrm{X}$ rods produces a maximum potential along the injection axis that is $9 \%$ of the applied RF potential. In the LCQ Deca, the maximum potential along the injection axis is $70 \%$ of the applied RF potential.

The efficiency of the ion injection process can be investigated by studying the effect of the RF amplitude during injection on the intensity of ions of various $\mathrm{m} / \mathrm{z}$. Figure 12a shows the dependence of the ion intensity for four different $\mathrm{m} / \mathrm{z}$ ions on the RF amplitude during injection for a 3-D ion trap. Figure 12b shows comparable data for the 2-D trap. The RF amplitude for this data is expressed in terms of the lowest stable $\mathrm{m} / \mathrm{z}$, i.e., the low mass cut-off (LMCO), and is directly proportional to the amplitude of the main RF. In comparing the data, it can be seen that the range of RF amplitudes that allows efficient trapping is quite limited in the 3-D trap, especially at lower $\mathrm{m} / \mathrm{z}$. This creates significant mass discrimination when using any single RF amplitude during ion injection. In contrast, the 2-D trap shows a significantly broader range of RF amplitudes that allows efficient trapping. Consequently, a single RF amplitude can be chosen to efficiently trap a wide mass range simultaneously, resulting in better sensitivity for full scan MS analysis. For example, an injection RF value corresponding to $100 \mathrm{LMCO}$ is used to efficiently and simultaneously trap a mass range of 150-2000 Da.

The phase dependence of ion injection into 3-D ion traps significantly limits the fraction of injected ions that are successfully trapped to less than 5\% [33]. Reports of trapping efficiencies as high as 100\% have been reported for linear traps due to the extended path length and reduction of RF fields along the axis of injection [3]. In our apparatus, ion current measurements indicated that the electrospray source and transfer optics deliver $40 \mathrm{pA}$ ( $2.5 \mathrm{e} 8$ charges/sec) through the front lens to the 2-D trap from the electrospray source. Ions were injected into the trap for 240 microseconds, for a total exposure of 60,000 charges. After ion injection, the contents of the ion trap were released to an

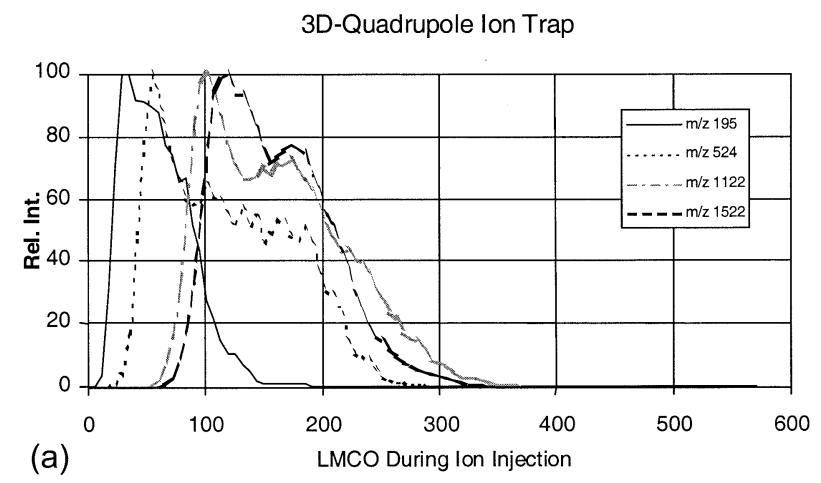

2D-Quadrupole Ion Trap

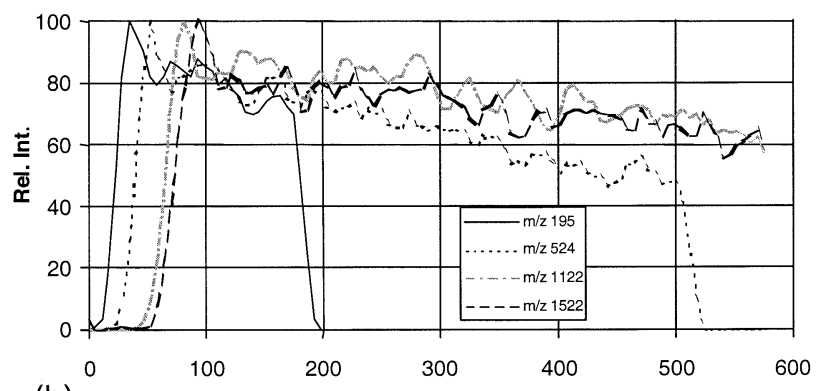

(b) LMCO During Ion Injection

Figure 12. Trapping efficiency dependence on the RF amplitude during ion injection for (a) 3-D and (b) 2-D ion traps. 


\section{Alprazolam - $500 \mathrm{fg}$ \\ MS/MS $309 \rightarrow 274$}

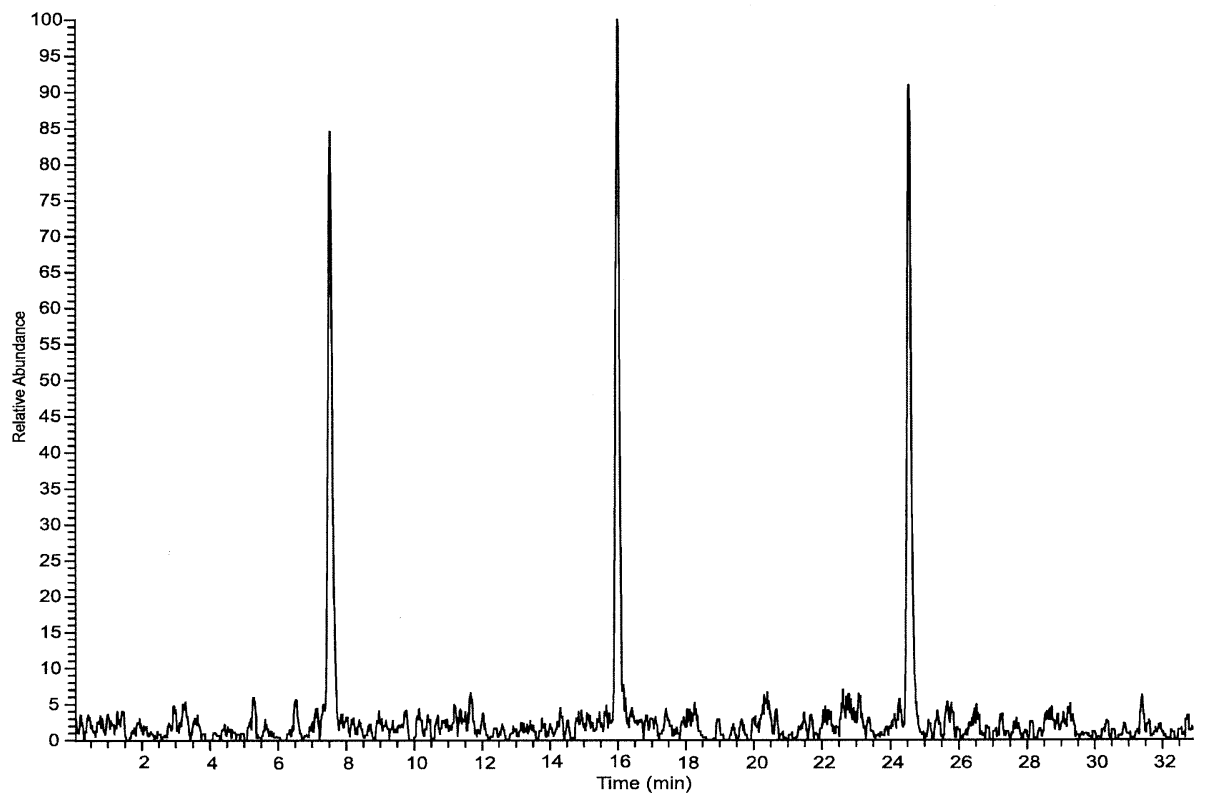

Figure 13. LC/MS/MS mass chromatogram for three injections of $500 \mathrm{fg}$ of alprazolam for the major fragment at $m / z 274$.

axial detector located to the rear of the ion trap. The accuracy of these determinations is limited by the accuracy of the measured gain of the detection system, which is better than $\pm 50 \%$. The current collected on this detector equated to approximately 17,400 charges, for an indicated accumulation efficiency of $29 \%$ for the injected ions, assuming no ion losses during the axial ejection. Although this is significantly less than previous work using 2-D ion traps, the difference may be explained by the use of a lighter collision gas and the much shorter trap length $(62 \mathrm{~mm})$. Successful ion accumulation is dependent on the ability to remove kinetic energy from an injected ion. A shorter trap and lighter collision gas means fewer beneficial collisions for removing this energy, and that each collision removes a smaller amount of kinetic energy. Both effects result in a reduced accumulation efficiency.

For a similar 240 microsecond injection, acquisition of a normal mass spectrum produced a total ion current equivalent to 7,600 charges. This produces a scan out efficiency of $44 \%$, or an overall detection efficiency of $12.7 \%$. It is assumed that half of the ions are neutralized on the rod opposite the ejection slot. The use of a second slot on the opposite rod in combination with a second detector should produce a scan out efficiency of $88 \%$, or an overall detection efficiency for ions injected into the trap of $\sim 25 \%$.

The trapping efficiency does depend on the pressure of buffer gas used and is compromised with resolution effects. The measured trapping efficiency of $29 \%$ indicates that the sensitivity of the 2-D trap should be approximately six times higher than the 3-D trap. This extra sensitivity can translate into lower detection limits especially for MS/MS analysis where chemical noise is eliminated. Figure 13 shows three replicate injections of 500 femtograms of alprazolam analyzed by LC/MS/MS $(\mathrm{m} / \mathrm{z} 309 \rightarrow 274)$. This is greater than a five times improvement over the detection limits of an LCQ Deca and seemed to roughly agree with the expected sensitivity advantage from the enhanced trapping efficiency.

\section{Future Directions and Dimensions}

The device used here was of limited length to allow the use of a standard detector. Longer 2-D ion traps have been used as storage devices with results that indicate the possibilities of higher ion capacity and trapping efficiency. Although the design of a new detector is not a major challenge, a potential difficulty of longer 2-D traps would be a reduction in mass spectral resolution due to the requirements of maintaining similar mechanical tolerances over a greater device length. As has been demonstrated, 2-D ion traps are readily incorporated into hybrid mass spectrometers since ion accumulation and ejection are both relatively efficient. The ability to produce independent mass spectral data in the 2-D trap portion of a trap-TOF [10] or trap-FTICR [7] provides the opportunity for far more sophisticated experiments than are currently possible.

\section{Conclusions}

A 2-D ion trap mass spectrometer using mass selective instability with resonance ejection has been constructed and its performance evaluated. This device is controlled and behaves much like a 3-D Paul-type ion trap mass 
spectrometer. Experiments thus far support the significant advantages of the 2-D trap for greater ion capacity or space charge limits and higher trapping efficiency of injected ions along with lower mass discrimination. Unit mass resolution at scan rates comparable to 3-D ion traps has been demonstrated along with high resolution spectra at reduced scan rates. Achieving useful resolution depends greatly on construction tolerances of the quadrupole structure and variation of end section potentials can be used to evaluate these tolerances. MS/MS has been demonstrated with isolation resolution and activation efficiencies which are also comparable to that of 3-D ion traps. This work sets the foundation to expand the use and technology of ion traps into new directions and dimensions.

\section{Acknowledgments}

The authors would like to acknowledge the assistance of numerous members of the scientific and engineering staff at Thermo Finnigan, including Paul Atherton, Wayne Dewey, Jim Gabel, Nigel Gore, Rex Heller, Eric Hemenway, Scott Quarmby, Alan Schoen, Bill Siebert, Berg Tehlirian and Rohan Thakur and also George Stafford and Ian Jardine for their support and encouragement of this project.

\section{References}

1. Prestage, J. D.; Dick, G. J. et al. New Ion Trap for Frequency Standard Applications. J. Appl. Phys. 1989, 66, 1013-1017.

2. Birkl, G.; Kassner, S. et al. Multiple-shell Structures of Lasercooled ${ }^{24} \mathrm{Mg}^{+}$Ions in a Quadrupole Storage Ring. Nature 1992, 357, 310-313.

3. Dolnikowski, G. G.; Kristo, M. J. et al. Ion-Trapping Technique for Ion/Molecule Reaction Studies in the Center Quadrupole of a Triple Quadrupole Mass Spectrometer. Int. J. Mass Spec. Ion Proc. 1988, 82, 1-15.

4. Senko, M. W.; Hendrickson, C. L. et al. External Accumulation of Ions for Enhanced Electrospray Ionization Fourier Transform Ion Cyclotron Resonance Mass Spectrometry. J. Am. Soc. Mass Spec. 1997, 8, 970-976.

5. Sannes-Lowery, K. A.; Griffey, R. H. et al. Multipole Storage Assisted Dissociation, a Novel In-source Dissociation Technique for Electrospray Ionization Generated Ions. Rapid Comm. Mass Spec. 1998, 12, 1957-1961.

6. Belov, M.; Nikolaev, E. N. et al. Design and Performance of an ESI Interface for Selective External Ion Accumulation Coupled to a Fourier Transform Ion Cyclotron Mass Spectrometer. Anal. Chem. 2001, 73, 253-261.

7. Syka, J. E. P.; Bai, D. L. et al. A Linear Quadrupole Ion Trap Fourier Transform Mass Spectrometer, A New Tool for Proteomics. 49th ASMS Conference on Mass Spectrometry and Allied Topics, Chicago, Illinois, 2001.

8. Ijames, C. F. A Proposed Two Dimensional Quadrupole/ Electrostatic Ion Trap Time-of-Flight Mass Spectrometer. 44th ASMS Conference on Mass Spectrometry and Allied Topics, Portland, Oregon, 1996.

9. Whitehouse, C. M.; Gulcicek, E. E. et al. A Two Dimensional Ion Trap API TOF Mass Spectrometer. 46th ASMS Conference on Mass Spectrometry and Allied Topics, Orlando, Florida, 1998.

10. Campbell, J. M.; Collings, B. A. et al. A New Linear Ion Trap Time-of-flight System with Tandem Mass Spectrometry Capabilities. Rapid Comm. Mass Spec. 1998, 12, 1463-1474.

11. Collings, B. A., Campbell, J. M. et al. A combined linear ion trap time-of-flight system with improved performance and MS $^{\mathrm{n}}$ capabilities. Rapid Comm. Mass Spec. 2001, 15, 1777-1795.
12. Douglas, D. J. Multipole Inlet System for Ion Traps. US Patent 5,179,278, 1991.

13. Cha, B.; Blades, M. W. et al. An Interface with a Linear Quadrupole Ion Guide for an Electrospray-Ion Trap Mass Spectrometer System. Anal. Chem. 2000, 72, 5647-5654.

14. Syka, J. E. P. and Fies, W. J. Jr. Fourier Transform Quadrupole Mass Spectrometer and Method. US Patent 4,755,670, 1988.

15. Bier, M. E. and Syka, J. E. P. Ion Trap Mass Spectrometer System and Method. US Patent 5,420,425, 1995.

16. Stafford, G. C.; Kelly, P. E. et al. Recent Improvements in and Analytical Applications of Advanced Ion Trap Technology. Int. J. Mass Spec. Ion Physics 1984, 60, 85-98.

17. Senko, M. W.; Schwartz, J. C. et al. Fourier Transform Mass Spectrometry in a Linear Quadrupole Ion Trap. 48th ASMS Conference on Mass Spectrometry and Allied Topics, Long Beach, California, 2000.

18. Welling, M.; Schuessler, H. A. et al. Ion/molecule reactions, mass spectrometry and optical spectroscopy in a linear ion trap. Int. J. Mass Spec. Ion Proc. 1998, 172, 95-114.

19. Lammert, S. A.; Plass, W.R.; Thompson, C. V.; Wise, M.B. Design, Optimization and Initial Performance of a Toroidal rf Ion Trap Mass Spectrometer. Int. J. Mass Spec. 2001, 88, 97-111.

20. Hager, J.W. A New Linear Ion Trap Mass Spectrometer. Rapid Comm. Mass Spec. 2002, 16, 512-526.

21. Beu, S. C.; Laude, D. A. J. Elimination of Axial Ejection during Excitation with a Capacitively Coupled Open Trapped-Ion Cell for Fourier Transform Ion Cyclotron Resonance Mass Spectrometry. Anal. Chem. 1992, 64, 177-180.

22. Syka, J. E. P. Commercialization of the Quadrupole Ion Trap. March, R. E.; Todd, J. F. J., Eds. Practical Aspects of Ion Trap Mass Spectrometry, Volume 1: Fundamentals of Ion Trap Mass Spectrometry, 1. CRC Press: Boca Raton, FL, 1995; 169-205.

23. Reiser, H. P.; Kaiser, R. E.; Savickas, P. J.; Cooks, R. G. Measurement of Kinetic Energies of Ions Ejected from a Quadrupole Ion Trap. Int. J. Mass Spec. Ion Proc. 1991, 106, 237-247.

24. Louris, J. N. and Taylor, D. M. Method and apparatus for ejecting unwanted ions in an ion trap mass spectrometer. US Patent 5,324,939, 1993.

25. Syka, J. E. P.; Louris, J. N. Method of operating ion trap detector in MS/MS mode. US Patent 4,736,101, 1988.

26. Moini, M. Ultramark 1621 as a Calibration/Reference Compound for Mass Spectrometry. II. Positive- and Negative-ion Electrospray Ionization. Rapid Commun. Mass Spec. 1994, 8, 711.

27. Schwartz, J. C. Do Space Charge Effects Limit LC Quadrupole Ion Trap Performance? 9th Sanibel Conference on Mass Spectrometry, Sanibel Island, Florida, 1997.

28. March, R. E.; Hughes, R. J. Quadrupole Storage Mass Spectrometry. Wiley: New York, 1989; 191.

29. Hemberger, P. H.; Nogar, N. S. et al. Laser Photodissociation Probe for Ion Tomography Studies in a Quadrupole Ion-trap Mass Spectrometer. Chem. Phys. Lett. 1992, 191, 405-410.

30. Schwartz, J. C.; Syka, J. E. P. et al. High Resolution on a Quadrupole Ion Trap Mass Spectrometer. J. Am. Soc. Mass Spec. 1991, 2, 198-204.

31. Schwartz, J. C.; Jardine, I. High Resolution Parent-ion Selection/Isolation Using a Quadrupole Ion-trap Mass Spectrometer. Rapid Commun. Mass Spec. 1992, 6, 313-317.

32. Louris, J. N.; Amy, J. W. et al. Injection of Ions into a Quadrupole Ion Trap Mass Spectrometer. Int. J. Mass Spec. Ion Proc. 1989, 88, 97-111.

33. Quarmby, S. T.; Yost, R. A. Fundamental Studies of Ion Injection and Trapping of Electrosprayed Ions on a Quadrupole Ion Trap. Int. J. Mass Spec. 1999, 190/191, 81-102. 